\title{
Bronchoscopic Cryotherapy for Acute Hypoxemic Respiratory Failure in Three Mechanically Ventilated Patients: A Case Series
}

\author{
Sudheer Tale ${ }^{1}$, Soibam P Meitei ${ }^{2}$, Vipul Prakash ${ }^{3}$, Arjun Negi ${ }^{4}$, Mayank Mishra ${ }^{5}$, Girish Sindhwani ${ }^{6}$
}

\begin{abstract}
Endobronchial blood clots or mucus plugs can present with minimal symptoms or acute airway obstruction in the intensive care unit (ICU) patients. Acute airway obstruction can lead to rapid worsening of dyspnea owing to poor oxygenation due to collapse of the lung. Prompt recognition and treatment of this condition can translate into a successful outcome by decreasing morbidity and mortality and facilitating successful weaning of these patients. When conventional methods fail to relieve the obstruction, cryoextraction a novel technique, may prove to be a useful alternative for the removal of these clots and mucus plugs. Cryoextraction is best performed with rigid bronchoscopic intubation. However, in certain conditions, it may be used with a flexible fiberoptic bronchoscope (FOB) through an endotracheal tube, especially when bedside procedure is required in ICU patients. In this series, three cases are being discussed where bedside flexible bronchoscopy-guided cryoextraction was done leading to a successful resolution of acute hypoxemic respiratory failure.

Keywords: Acute airway obstruction, Acute hypoxemic respiratory failure, Cryoextraction, Fiberoptic bronchoscopy, Intensive care unit, Lung collapse.

Indian Journal of Critical Care Medicine (2021): 10.5005/jp-journals-10071-23707
\end{abstract}

\section{INTRODUCTION}

Acute hypoxemic respiratory failure is one of the main indications for admission of critically ill patients to the intensive care unit (ICU). In a proportion of these patients, acute airway obstruction can occur due to mucus plugging, central tumors, or blood clots. Trauma patients with blunt injury to the chest, patients with hemoptysis, and other patients with chronic respiratory diseases like asthma with allergic bronchopulmonary aspergillosis (ABPA) may get admitted with hypoxemia secondary to complications like airway obstruction with lung collapse. Prompt clinical examination and chest radiographs can help the clinician to diagnose these conditions. Traditional methods of relieving such obstruction involve the use of normal saline flush, suction, and forceps passed through the working channel of the bronchoscope. However, the procedure is often a tedious one.

Cryoextraction is the procedure wherein a cryoprobe is passed down the working channel of the bronchoscope to freeze the blood clots or mucus plugs and the cryoprobe is withdrawn along with the bronchoscope as a whole. ${ }^{1}$ This method allows the clinician to remove culprit mucus plugs or blood clots much more efficiently and rapidly than would be possible with conventional methods since it allows en bloc removal without much risk for breakage into smaller pieces. Hence, these conditions are the common situations wherein fiberoptic bronchoscope (FOB) cryotherapy is indicated in the ICU setting to facilitate rapid resolution of airway obstruction. ${ }^{2}$

Moreover, the development of a thin and flexible cryoprobe (1.9 and $2.4 \mathrm{~mm}$ ) has allowed bronchoscopic cryotherapy to be performed easily in mechanically ventilated patients without any significant risk of hypoxemia. ${ }^{3}$ Contraindications to bronchoscopic cryotherapy will be the usual contraindications to bronchoscopy including hemodynamic instability, myocardial ischemia, lifethreatening arrhythmias, and lack of informed consent. ${ }^{4}$
${ }^{1-4,6}$ Department of Pulmonary and Critical Care Medicine, All India Institute of Medical Sciences, Rishikesh, India

${ }^{5}$ Department of Pulmonary Medicine, All India Institute of Medical Sciences, Dehradun, Uttarakhand, India

Corresponding Author: Sudheer Tale, Department of Pulmonary and Critical Care Medicine, All India Institute of Medical Sciences, Rishikesh, India, Phone: +91 8872156777, e-mail: drtalesudheer@gmail.com

How to cite this article: Tale S, Meitei SP, Prakash V, Negi A, Mishra M, Sindhwani G. Bronchoscopic Cryotherapy for Acute Hypoxemic Respiratory Failure in Three Mechanically Ventilated Patients: A Case Series. Indian J Crit Care Med 2021;25(1):94-96.

Source of support: Nil

Conflict of interest: None

Here, we report three different cases in which bedside flexible bronchoscopic cryoextraction was performed leading to a successful resolution of hypoxemia and subsequent weaning from mechanical ventilation.

\section{Case Descriptions}

\section{Case 1}

A 42-year-old woman with a history of cough with expectoration, dyspnea, and intermittent fever for 2 years, presented to the emergency with acute worsening of breathlessness for 2 days. She had received antitubercular treatment (ATT) twice in the past without any improvement and was using short-acting beta- 2 agonists for reactive airway disease. On examination, she was febrile, tachypneic, with reduced breath sounds over left lung fields and $\mathrm{SpO}_{2}$ of $60 \%$ on room air. She was intubated, shifted to ICU, and started on mechanical ventilation. The chest radiograph showed left lung collapse (Fig. 1A). Bedside diagnostic FOB revealed thick mucus 
plugs causing complete occlusion of the left mainstem bronchus. A 2.4-mm cryoprobe (ERBE CRYO-2 Cart, Tübingen, Germany) under flexible FOB guidance was used to remove these mucus plugs in two sessions. Allergic bronchopulmonary aspergillosis workup was remarkable for elevated levels of Aspergillus-specific lgE (13.6 $\mathrm{kUA} / \mathrm{L})$, total $\lg \mathrm{E}(2,307 \mathrm{IU} / \mathrm{mL})$, and eosinophil count (630 cells/ $\mathrm{mm}^{3}$ ). Keeping "Severe asthma with ABPA" as the most probable diagnosis, she was started on systemic corticosteroids. There was gradual clinical and radiographic (Fig. 1B) improvement and later weaned off from mechanical ventilation and discharged in stable condition. Since then she has been in regular follow-up with us and was doing well till her last visit. We went for bronchoscopic cryoextraction in this patient since the multiple large thick mucus plugs in the left main bronchus (LMB) were difficult to remove by conventional techniques.

\section{Case 2}

A 52-year-old man presented to the emergency with a bout of hemoptysis of approximately more than $300 \mathrm{~mL}$. He had a past history of pulmonary tuberculosis for which he had taken ATT 4 years ago. After initial resuscitation and stabilization in the emergency department, he was planned for bronchial artery embolization (BAE).

Computed tomography aortogram revealed dilated tortuous bronchial arteries and fibro-bronchiectatic changes in both the

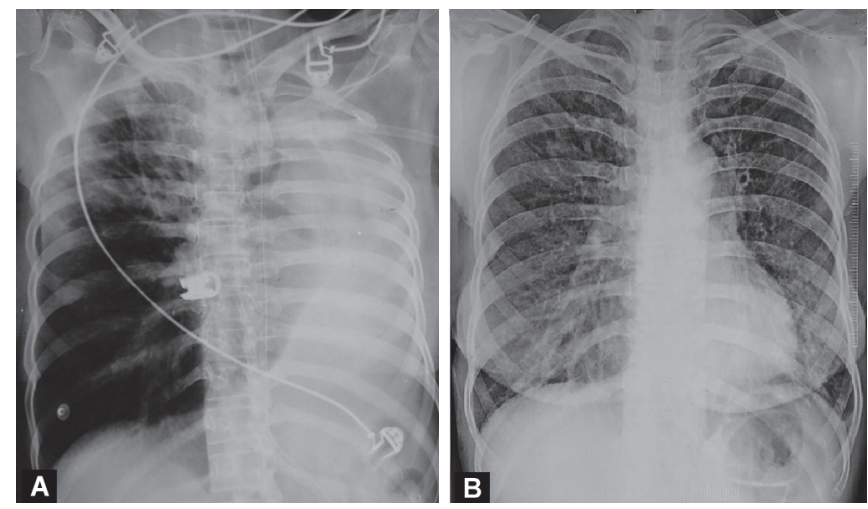

Figs $1 \mathrm{~A}$ and $\mathrm{B}$ : $(\mathrm{A})$ Preprocedure chest radiograph depicting left lung collapse; (B) Follow-up chest radiograph revealed well-aerated left lung

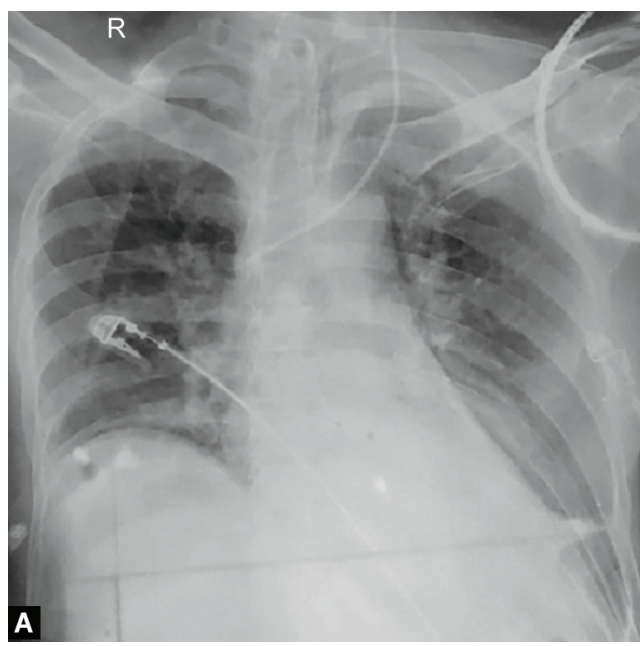

lungs. Selective embolization of tortuous left bronchial artery was done and the patient was shifted to ICU, where he had a bout of massive hemoptysis again needing endotracheal intubation. Bedside chest radiograph showed right middle and lower lobe collapse (Fig. 2A). Urgent bedside FOB revealed a thick large blood clot snugly fitting into the bronchus intermedius, preventing any further advancement of the bronchoscope. Multiple attempts at removing the clot with suction proved unsuccessful, which was then removed successfully using a $2.4-\mathrm{mm}$ cryoprobe. Post-procedure chest radiograph showed a return of good aeration (Fig. 2B). Hemoptysis was controlled after performing repeat $B A E$, and the patient was weaned off from the ventilator. Thus, bronchoscopic cryoextraction helped in the en bloc removal of the thick blood clots, which was difficult to be achieved with conventional techniques.

\section{Case 3}

A 32-year-old man with an alleged history of road traffic accident (RTA) was admitted to the ICU with multiple injuries needing intubation and mechanical ventilation. His initial chest radiograph was normal. On day 3 of the ICU stay, he developed tachycardia and increased oxygen requirement. Bedside chest radiograph was suggestive of left lung lower lobe collapse (Fig. 3A). Diagnostic bedside bronchoscopy revealed a large blood clot completely occluding the left lower lobe bronchus. It was successfully removed by a $2.4-\mathrm{mm}$ cryoprobe (Fig. 3B) following


Figs $2 A$ and B: (A) Preprocedure chest radiograph of the patient showing right middle and lower lobe collapse; (B) Follow-up chest radiograph showed well-aerated bilateral lung zones

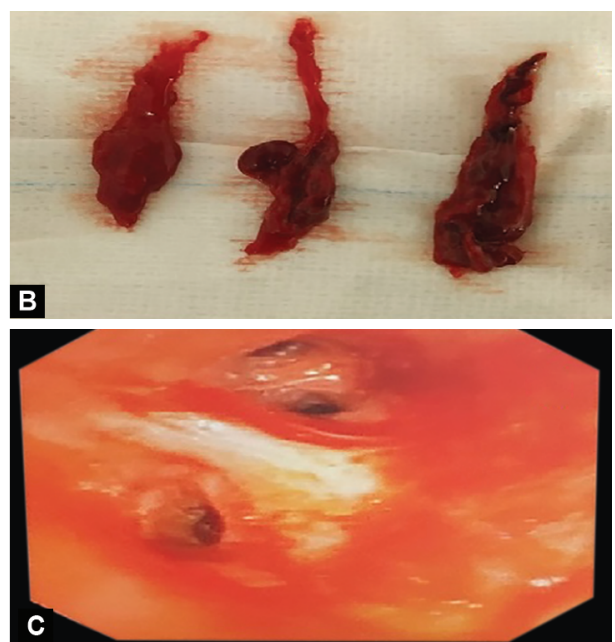

Figs 3A to C: (A) Chest radiograph showing left lower lobe collapse; (B) Image showing blood clots removed by cryoprobe; (C) Image depicting patent left lower lobe bronchus after removal of blood clots 
which his chest radiograph improved with the return of aeration. In view of the prolonged need for mechanical ventilation, he was tracheostomized and shifted to the ward for the management of orthopedic injuries. In this patient, cryotherapy led to prompt removal of thick blood clots and therefore rapid improvement in the clinical condition of the patient.

\section{Discussion}

Acute airway obstruction due to mucus plugs, blood clots, and tumors can occur in various clinical conditions like bronchiectasis, hemoptysis, blunt trauma to the chest, and in malignant cases. ${ }^{5}$ These conditions may present with life-threatening hypoxemia leading to rapid clinical deterioration if the airway obstruction is not diagnosed promptly. After initial stabilization, various general measures like nebulization, chest physiotherapy, mucolytics, and ET suction (chosen as per the indication) may help relieve such an obstruction in some cases. Bronchoscopic cryoextraction comes into play if general measures fail to improve the clinical condition.

Cryotherapy employs extremely low temperatures to freeze the tissue for adhesion (cryoadhesion), destruction (cryoablation), or biopsy (cryobiopsy). ${ }^{6,7}$ The principle of cryotherapy is based on the Joule Thomson effect in which a liquefied gas under pressure when exits through a small orifice undergoes rapid conversion and expansion to the gaseous form. This liquid-gas conversion is accompanied by a dramatic temperature drop that is captured at the tip of the cryoprobe. During cryoextraction, tissues are frozen at the tip of the cryoprobe and removed along with the probe and bronchoscope, all as a single unit. ${ }^{2}$

Conventional bronchoscopic methods for removal of blood clots or mucus plugs usually result in incomplete patency of the airway. ${ }^{8}$ Moreover, these procedures are time-consuming which might prove hazardous for a critically ill patient on mechanical ventilation. Cryoextraction is a very useful tool that enables rapid removal of mucus plugs or blood clots in a single or few steps. ${ }^{9}$ Another advantage of using cryotherapy in critically ill patients is that it does not require a reduction of $\mathrm{FiO}_{2}$ during the procedure. Cryoprocedures are best performed through a rigid bronchoscope, although it can be done by flexible bronchoscope through ET tube to reduce morbidity and mortality associated with rigid bronchoscopy in sick patients. One limitation about using flexible FOB-guided cryoprobe removal of endobronchial blood clots is that a large blood clot might be difficult to extract through the small-sized ET. In such a situation, the ET tube has to be removed along with the blood clot and then reinserted. Cryotherapy is safe for the user and the patient is compatible with oxygen and is a safer alternative for mechanically ventilated and sick patients. In all three of our critically ill mechanically ventilated patients, we successfully used flexible FOB with a 2.4-mm cryoprobe for removal of mucus plugs and clots through ET. The use of cryoextraction for the removal of endobronchial blood clots has previously been reported by some authors. ${ }^{10,11}$

However, to the best of our knowledge, this is the first case series in India wherein cryotherapy has been used for the removal of mucus plugs and blood clots.

\section{References}

1. Franke KJ, Nilius G, Rühle KH. Use of cryoextraction in different types of airway obstruction. Pneumologie. 2010;64(6):387-389. DOI: 10.1055/s-0029-1244046.

2. Sriratanaviriyakul N, Lam F, Morrissey BM, Stollenwerk N, Schivo M, Yoneda KY. Safety and clinical utility of flexible bronchoscopic cryoextraction in patients with non-neoplasm tracheobronchial obstruction: a retrospective chart review. J Bronchology Interv Pulmonol 2015;22(4):288-293. DOI: 10.1097/LBR.0000000000000203.

3. De Weerdt S, Noppen M, Remels L, Vanherreweghe R, Meysman M, Vincken W. Successful removal of a massive endobronchial blood clot by means of cryotherapy. J Bronchol 2005;12(1):23-24. DOI: 10.1097/00128594-200501000-00007.

4. Mohan A, Madan K, Hadda V, Tiwari P, Mittal S, Guleria R, et al. Guidelines for diagnostic flexible bronchoscopy in adults: Joint Indian Chest Society/National College of Chest Physicians (I)/ Indian Association for Bronchology recommendations. Lung India 2019;36(8):S37-S89. DOI: 10.4103/lungindia.lungindia_108_19.

5. Schmidt LH, Schulze AB, Goerlich D, Schliemann C, Kessler T, Rottmann $\mathrm{V}$, et al. Blood clot removal by cryoextraction in critically ill patients with pulmonary hemorrhage. J Thorac Dis 2019;11(10):4319-4327. DOI: 10.21037/jtd.2019.09.46.

6. Beamis JF, Becker HD, Cavaliere S, Colt H, Diaz-Jimenez JP, Dumon $J F$, et al. ERS/ATS statement on interventional pulmonology. European Respiratory Society/American Thoracic Society. Eur Respir J 2002;19(2):356-373. DOI: 10.1183/09031936.02.00204602.

7. Dhooria S, Agarwal R, Sehgal I, Aggarwal A, Goyal R, Guleria R, et al. Bronchoscopic lung cryobiopsy: An Indian Association for Bronchology Position Statement. Lung India 2019;36(1):48-59. DOI: 10.4103/lungindia.lungindia_75_18.

8. Cetin G, Corut R, Kupeli E. Removal of a large endobronchial blood clot using a flexible bronchoscope. J Bronchology Interv Pulmonol 2012;19(4):358-359. DOI: 10.1097/LBR.0b013e31826ca6a5.

9. Lee $H$, Leem CS, Lee JH, Lee CT, Cho YJ. Successful removal of endobronchial blood clots using bronchoscopic cryotherapy at bedside in the intensive care unit. Tuberc Respir Dis 2014;77(4):193196. DOI: 10.4046/trd.2014.77.4.193.

10. Sehgal IS, Dhooria S, Agarwal R, Behera D. Use of a flexible cryoprobe for removal of tracheobronchial blood clots. Respiratory Care 2015;60(7):e128-e131. DOI: 10.4187/respcare.03861.

11. Bhardwaj B, Bhardwaj H, Youness HA, Awab A. Bronchoscopic cryoextraction: a novel approach for the removal of massive endobronchial blood clots causing acute airway obstruction. South west J Pulm. Crit Care 2013;7(3):184-189. DOI: 10.13175/swjpcc112-13. 